\title{
Riesgo de impactación temprana de caninos maxilares en radiografías panorámicas en las clínicas de la Universidad Santo Tomás, Bucaramanga
}

\author{
Risk Early impaction of maxillary canines in clinical panoramic radiographs \\ Universidad Santo Tomás, Bucaramanga
}

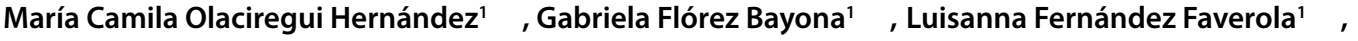 \\ María Camila Castellanos Rodríguez ${ }^{1}$ (D) , Adriana Esperanza Peñuela Sánchez ${ }^{2}$ (D), Ethman Ariel Torres Murillo ${ }^{3}$ (D)
}

Citación: Olaciregui MC, Flórez G, Fernández L, Castellanos MC, Peñuela AE, Torres E. Riesgo de impactación temprana de caninos maxilares en radiografías panorámicas clínicas Universidad SantoTomás, Bucaramanga. Ustasalud 2022;21(1):17-24. DOI: https://doi.org/10.15332/us.v21i1.2584

Licencia Creative Commons

\section{(c) $(1)(9)$} alterarlos, adicionalmente se debe reconocer la autoría de las personas que figuran en las publicaciones, pero estas no podrán ser comercializadas.

\section{Resumen}

Objetivo: Determinar el riesgo de impactación temprana de caninos maxilares por medio de la radiografía panorámica en niños etapa dentición mixta que asistieron a las clínicas de la Universidad Santo Tomás de Floridablanca en el periodo del 2018 a 2020. Materiales y métodos: Se realizó un estudio observacional analítico de corte transversal, analizando las variables: edad, sexo; etapa dentición mixta y estadio de Nolla del canino maxilar; para determinar el riesgo de impactación del canino maxilar; utilizando la radiografía panorámica se utilizó el método de Ericson y Kurol, en el que; si el canino estaba en posición 4 o 5, el ángulo alfa era mayor a $25^{\circ}$, el ángulo beta era mayor a $35,6^{\circ}$ o la distancia de la cúspide del canino al plano oclusal era mayor a 18,46 $\mathrm{mm}$ se consideraba que el diente estaba en riesgo de impactación temprana. Este estudio fue clasificado como investigación sin riesgo y fue aprobado por el Comité de Ética de la Universidad Santo Tomás. Resultados: Se evaluaron 171 radiografías de niños y niñas con edades de 9,3 $\pm 1,1$ años; el 56,7\% de las participantes eran niñas. El estadio de Nolla más frecuente en la investigación fue de formación radicular de $2 / 3$; con un $47,6 \%$ y la posición según Ericson y Kurol fue la 1 en un $89,4 \%$. Los niños que presentaron riesgo de impactación tenían en promedio 9,7 $\pm 1,0$ años. La mayoría de dientes con riesgo de impactación estaban en el segundo periodo transicional con un $51,7 \%$ y el no riesgo de impactación estaban en el periodo intertransicional con un $66,2 \%(p=0,010)$. Conclusiones: El riesgo de impactación temprana de caninos maxilares se diagnosticó en el 10,8\% de los caninos evaluados por medio de la radiografía panorámica, lo que indica la necesidad de un diagnóstico temprano.

Palabras clave: Diente impactado, diente canino, radiografías panorámicas.

\begin{abstract}
Objective: To determine the risk of early impaction of maxillary canines through panoramic radiography in children with mixed dentition stage who attended the Santo Tomás de Floridablanca University clinics in the period from 2018 to 2020. Materials and methods: An analytic study was carried out, descriptive cross section; analyzing the variables: age, sex; mixed dentition stage and nolla stage of maxillary canine; to determine the risk of impaction of the maxillary canine; using panoramic radiography, the Ericson and Kurol method was used; in which, if the canine was in position 4 or 5 , the alpha angle was greater than $25^{\circ}$, the beta angle was greater than $35.6^{\circ}$ or the distance from the cusp of the canine to the occlusal plane was greater than $18.46 \mathrm{~mm}$, the tooth was considered to be at risk for early impaction. This study was classified as riskfree research and was approved by the Santo Tomás University Ethics Committee. Results: 171 X-rays of boys and girls aged $9.3 \pm 1.1$ years were evaluated; $56.7 \%$ of the participants were girls. The most frequent nolla stage in the investigation was $2 / 3$ root formation; with $47.6 \%$ and the position according to Ericson and Kurol was 1 in $89.4 \%$. The children who were at risk of impaction were $9.7 \pm 1.0$ years old on average. The majority of teeth at risk of impaction were in the second transitional period with $51.7 \%$ and those without risk of impaction were in the intertransitional period with $66.2 \%(p=0.010)$. Conclusions: The risk of early impaction of maxillary canines was diagnosed in $10.8 \%$ of the canines evaluated by means of panoramic radiography, which indicates the need for an early diagnosis.
\end{abstract}

Keywords: Impacted tooth, canine tooth, panoramic radiographs.
1 Estudiante de Odontología, Universidad Santo Tomás Seccional Bucaramanga. Colombia.

2 Médico veterinario. Magíster en Epidemiología. Universidad Santo Tomás Seccional Bucaramanga. Colombia

3 Odontológo, Especialista en Estomatología Pediátrica y en Ortodoncia. Universidad Santo Tomás Seccional Bucaramanga, Colombia

Autor de correspondencia:

María Camila Olaciregui Hernández Correo electrónico:

maria.olaciregui@ustabuca.edu.co
Recibido para publicación 22 de enero 2021 Aceptado para publicación: 13 de julio 2021 


\section{INTRODUCCIÓN}

La alteración de erupción conocida como impactación está caracterizada por la imposibilidad que tiene el diente para erupcionar en una posición correcta; se pueden presentar por diversas causas, pero la más común está relacionada con la falta de espacio y alteración de la secuencia de erupción [1]. Los caninos maxilares son los dientes responsables del mayor crecimiento transversal del maxilar superior; consolidando las relaciones transversales y sagitales de la oclusión para obtener una alineación y simetría del arco con relaciones caninas clase I; un paciente sin erupción adecuada de caninos tendrá disminución de distancia intercanina y perímetro de arco, alteración de sus relaciones oclusales y asimetría de línea media; por ello, el clínico que atiende pacientes en etapa dentición mixta debe vigilar y controlar la erupción adecuada de los caninos; en cronología y secuencia de su erupción; para asegurar un correcto desarrollo del arco maxilar y por ende de todo el crecimiento craneofacial [1-2].

Los caninos permanentes maxilares son los dientes que presentan mayor prevalencia en alteraciones de la vía eruptiva; se considera normal que este diente este erupcionando entre los 11 y 13 años de edad; con diferencias según población y sexo; primero erupcionan en niñas que en niños [2]. Moyers resalta que al ser los caninos los últimos dientes permanentes en erupcionar en la zona anterior, es común observar múltiples alteraciones en su erupción [3].

La impactación de caninos ocasiona alteraciones entre las que se destacan: malposición lingual o labial del diente retenido, migración del diente vecino y pérdida de la longitud de arco, reabsorción interna, formación de quistes dentígeros, reabsorción radicular externa del canino retenido, reabsorción radicular externa de los dientes vecinos, infección relacionada con la erupción parcial, dolor referido y combinación de alteraciones en la oclusión que altera la estética y función [4-5].

Para determinar el riesgo de la impactación de los caninos se emplean las radiografías bidimensionales, más específicamente las panorámicas, estas permiten la evaluación de riesgo; control del proceso de erupción, diagnóstico temprano y seguimiento del tratamiento correspondiente [5-7]. En 1986 Ericson y Kurol realizaron un estudio longitudinal que mostró una prevalencia del 7\% de la

posibilidad de impactación de caninos maxilares y la necesidad del seguimiento en estos pacientes [8]. Se resalta la importancia del tratamiento preventivo para evitar las graves complicaciones de un canino impactado; este tratamiento preventivo se debe hacer de manera clínica y con ayudas diagnósticas, como la radiografía panorámica o la tomografía; se debe realizar una valoración que incluya anamnesis e historia clínica, observando y palpando; además de los exámenes necesarios según cada caso particular y un cuestionamiento de antecedentes familiares y personales [8].

Diversas investigaciones han mostrado la importancia de la detección temprana del riesgo de caninos impactados; utilizando métodos de diagnóstico que incluye tanto radiografías panorámicas como tomografías; dentro de las más relevantes encontramos los estudios de Alqerban y colaboradores [9] que muestran la efectividad de la radiografía panorámica para detectar tempranamente la impactación de los caninos maxilares. Al no contar en las clínicas de la universidad con un estudio en este tema y teniendo presente la importancia de enseñar la detección temprana de esta alteración; se hace este trabajo con el objetivo de determinar el riesgo de impactación temprana de los caninos maxilares mediante el empleo de la radiografía panorámica en los pacientes que asisten a las Clínicas de la Universidad Santo Tomás, Campus Floridablanca, en el periodo de 2018 a 2020.

\section{MATERIALES Y MÉTODOS}

Se llevó a cabo un estudio observacional analítico de corte transversal, con tamaño de muestra de 171 radiografías panorámicas, pertenecientes al archivo digital de la Clínica de Pediatría de la Universidad Santo Tomás, y que fueron seleccionadas de los pacientes en etapa de dentición mixta, tomadas en los años 2018-2020. Se hizo un muestreo por conveniencia, excluyendo radiografías que tuvieran agenesia de caninos, imágenes no claras o superpuestas sin calidad, que no permitieran hacer trazos digitales, también se excluyeron las radiografías que en su historia clínica no tuvieran el consentimiento informado firmado. 
En el estudio se incluyeron variables sociodemográficas (edad y sexo); etapa dentición mixta del paciente (primer periodo; periodo intertransicional; segundo periodo); grado de formación radicular según estadio de Nolla. Las medidas utilizadas para determinar el riesgo de impactación del canino maxilar se tomaron del método publicado por Ericson y Kurol para radiografías panorámicas [8]: posición mesodistal de la corona, inclinación del canino en relación con la línea media, inclinación con relación al incisivo lateral, la identificación del grado de erupción del canino y, finalmente, el análisis de riesgo de impactación. El método consta de "establecer 5 ejes longitudinales del incisivo central e incisivo lateral (permanentes) y líneas paralelas que pasan por el punto de contacto entre centrales, lateral con central, mesial y distal del canino deciduo" (Figura 1).

Se evalúo el ángulo a el cual es el eje longitudinal del canino con la línea media, donde obtener un ángulo de $25^{\circ}$ es normal, si es mayor el diente presentará riesgo de impactación según este criterio; también se evaluó el grado de erupción vertical del canino (d1) el cual es registrado con la distancia en milímetros de la cúspide del canino con el plano oclusal (Figura 2) [8-9].

También se determina el ángulo $\beta$, el cual se toma trazando el eje longitudinal del incisivo lateral permanente con el eje longitudinal del canino en erupción (Figura 3) [9].

Estas mediciones se realizaron en forma digital por un técnico en radiología odontológica; se hizo una calibración interexaminador con un experto y se calculó el Coeficiente Kappa de Cohen para obtener un valor de 0,93 para el total de las cuatro mediciones tomadas de forma digital; los datos fueron registrados en dos bases de datos creados para tal fin. Estas fueron validadas con el software Epidata 3,1 y los errores de digitación fueron corregidos con los instrumentos. Una base completamente depurada fue exportada al programa estadístico Stata I/C versión 14.

Se hizo un análisis univariado, en el que se presentaron medidas de resumen de acuerdo con la naturaleza de las variables. El análisis bivariado estudió las diferencias entre variables cualitativas, por medio de la prueba Chi cuadrado o el Test Exacto de Fisher.
También se hicieron comparaciones con variables cuantitativas usando las pruebas $\mathrm{T}$ de Student o $\mathrm{U}$ de Mann-Whitney. Para todas las pruebas se consideró significancia estadística para aquellos valores $\mathrm{P}<0,05$.

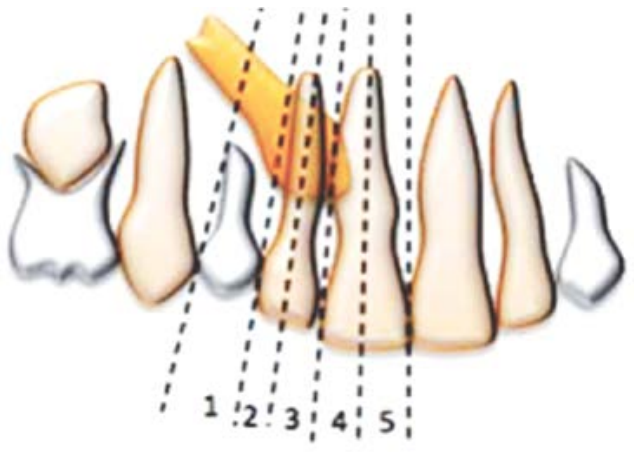

Figura 1. Método Ericson y Kurol para determinar la posición mesodistal de la corona del canino. Fuente: [8].

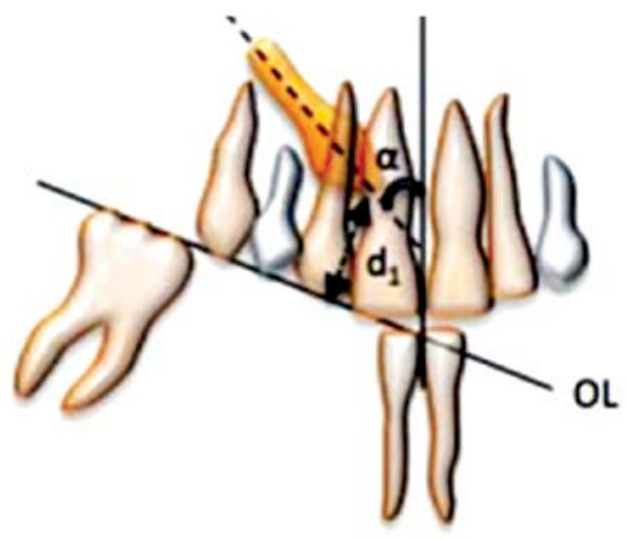

Figura 2. Trazado para determinar la inclinación del canino con la línea media [8] y medida d1. Fuente: [8].

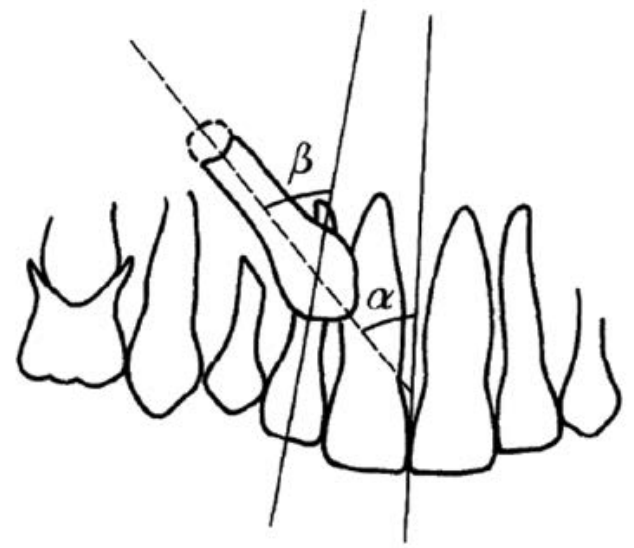

Figura 3. Trazado para calcular angulación del canino con el eje longitudinal del diente lateral. Fuente: [9]. 
Este trabajo se realizó según las consideraciones éticas actuales y vigentes. Se tuvo en cuenta la normatividad contemplada en la resolución No. 08430 de 1993, según la cual, de acuerdo con el artículo 11, el presente estudio se consideró como investigación sin riesgo.

\section{RESULTADOS}

Como se observa en la Tabla 1 de las 171 radiografías evaluadas, el 56,7\% correspondía a mujeres y el promedio de edad fue de 9,3 $\pm 1,1$ años. El periodo de dentición mixta más relevante fue el intertransicional con $61,9 \%$.

Tabla 1. Descripción de datos sociodemográficos de los pacientes que asistieron a las clínicas de la USTA

\begin{tabular}{lc}
\hline Variable & $\mathbf{n}(\%)$ \\
\hline Edad & $9,3^{*}(1,1)^{* *}$ \\
\hline Sexo & $97(56,7)$ \\
\hline Femenino & $74(43,2)$ \\
\hline Masculino & \\
\hline Periodo de dentición mixta & $106(61,9)$ \\
\hline Intertransicional & $62(36,2)$ \\
\hline Segundo periodo transicional & $3(1,7)$ \\
\hline Primer periodo transicional & \\
\hline${ }^{*}$ Media ${ }^{* *}$ Desviación estándar. & \\
Fuente: Autores.
\end{tabular}

En total se evaluaron 342 caninos maxilares, de los cuales el $89,4 \%$ se encontraba en posición dental 1 . De esos caninos predominó el estadío de Nolla 8 , el cual es $2 / 3$ de formación de raíz, con $47,6 \%$ (Tabla 2).

Tabla 2. Descripción de características específicas de los caninos maxilares evaluados en radiografías panorámicas

\begin{tabular}{lc}
\hline Posición de la pieza dental (Figura 1$)$ & $\mathbf{n}(\%)$ \\
\hline 1 Posición adecuada de canino & $\mathbf{3 0 6}(\mathbf{8 9 , 4 )}$ \\
\hline 2 Canino sobrepasa mitad distal del lateral & $\mathbf{3 4 ( 9 , 9 )}$ \\
\hline 3 Canino sobrepasa todo el lateral & $\mathbf{2 ( 0 , 5 )}$ \\
\hline Estadío de Nolla & \\
\hline Corona completa; estadío 6 & $\mathbf{5 ( 1 , 4 6 )}$ \\
\hline 1/3 de raíz; estadío 7 & $\mathbf{1 1 5 ( 3 3 , 6 )}$ \\
\hline 2/3 de raíz; estadío 8 & $\mathbf{1 6 3 ( 4 7 , 6 )}$ \\
\hline Raíz casi completa; estadío 9 & $\mathbf{5 6}(\mathbf{1 6 , 3 )}$ \\
\hline Raíz completa y ápice cerrado estadío 10 & $\mathbf{3 ( 0 , 8 )}$ \\
\hline
\end{tabular}

Fuente: Autores.
Se encontraron 29 radiografías con al menos 1 diente con riesgo temprano de impactación, lo que correspondió a $16,9 \%$ de estas 29 radiografías; 21 radiografías panorámicas tuvieron un solo diente con riesgo temprano de impactación y 8 presentaron los dos dientes con el riesgo temprano de impactación. En cuanto al total de caninos evaluados, el 10,8\% presentaron riesgo temprano de impactación.

Se observaron diferencias en cuanto a la edad, ya que los niños que presentaron riesgo temprano de impactación tenían en promedio 9,7 $\pm 1,0$ años $(\mathrm{p}=0,059)$. También se observaron diferencias en cuanto al tipo de dentición, ya que la mayoría de los dientes con riesgo temprano de impactación se encontraba en el segundo periodo transicional con un $51,7 \%(\mathrm{p}=0,010)$. En cuanto al género no se encontraron diferencias estadísticamente significativas (Tabla 3 ).

Tabla 3. Riesgo de impactación relacionado con datos sociodemográficos

\begin{tabular}{lccc}
\hline \multicolumn{1}{c}{ Variable } & $\begin{array}{c}\text { Riesgo de } \\
\text { impactación } \\
\mathbf{n}(\%)\end{array}$ & $\begin{array}{c}\text { No } \\
\text { impactación } \\
\mathbf{n}(\%)\end{array}$ & Valor $\mathbf{p}$ \\
\hline Edad & $9,7^{*}(1,0) \ddagger$ & $9,3^{*}(1,1) \ddagger$ & $0,059 \S$ \\
\hline Sexo & $16(55,1)$ & $81(57,0)$ & $0,853^{* *}$ \\
\hline Femenino & $13(44,8)$ & $61(42,9)$ & \\
\hline Masculino & & & $0,010 \|$ \\
\hline Dentición & $12(41,3)$ & $94(66,2)$ & \\
\hline Intertransicional & $15(51,7)$ & $47(33,1)$ & \\
\hline $\begin{array}{l}\text { Segundo periodo } \\
\text { transicional }\end{array}$ & $2(6,9)$ & $1(0,7)$ & \\
\hline Dentición mixta & & & \\
\hline
\end{tabular}

${ }^{*}$ Media $\ddagger$ Desviación estándar $₫$ Valor $p$ de prueba $t$ de Student ${ }^{*}$ Valor $p$ prueba Chi $i^{2}$ |Valor p prueba Fisher's exact.

Fuente: Autores.

En la Tabla 4 se muestra diferencia en la posición dental donde los caninos con riesgo temprano de impactación se encontraban en la posición 1 con un $78,3 \%(\mathrm{p}=0,030)$. Por otra parte, en el estadío de Nolla no se encontró diferencia estadística. 
Tabla 4. Riesgo de impactación de caninos en relación con características específicas de los caninos maxilares

\begin{tabular}{lccc}
\hline \multicolumn{1}{c}{ Variable } & $\begin{array}{c}\text { Riesgo de } \\
\text { impactación } \\
\mathbf{n}(\%)\end{array}$ & $\begin{array}{c}\text { No } \\
\text { impactación } \\
\mathbf{n}(\%)\end{array}$ & $\begin{array}{c}\text { Valor } \\
\text { p } \|\end{array}$ \\
\hline Estadio de Nolla & & & 0,285 \\
\hline Corona completa & $1(2,7)$ & $4(1,3)$ & \\
\hline $1 / 3$ de raíz & $15(40,5)$ & $100(32,7)$ & \\
\hline $2 / 3$ de raíz & $16(43,2)$ & $147(48,2)$ & \\
\hline Raíz casi completa & $4(10,8)$ & $51(17,0)$ & \\
\hline $\begin{array}{l}\text { Raíz completa y } \\
\text { ápice cerrado }\end{array}$ & $1(2,7)$ & $2(0,66)$ & \\
\hline $\begin{array}{l}\text { Posición dental } \\
\text { (Figura } 1)\end{array}$ & & & \\
\hline 1 & $29(78,3)$ & $277(90,8)$ & \\
\hline 2 & $7(18,9)$ & $27(8,8)$ & \\
\hline 3 & $1(2,7)$ & $1(0,3)$ & \\
\hline
\end{tabular}

|| Valor p prueba Fisher's exact.

Fuente: Autores.

Se muestra una diferencia estadísticamente significativa para las mediciones de los ángulos y la distancia, siendo los tres valores más altos para los caninos con riesgo temprano de impactación comparados con los caninos no impactados (Tabla 5).

Tabla 5. Riesgo de impactación de caninos relacionado con mediciones propuestas por Ericson y Kurol

\begin{tabular}{lccc}
\hline \multicolumn{1}{c}{ Variable } & $\begin{array}{c}\text { Riesgo de } \\
\text { impactación } \\
\text { Media (DE) }\end{array}$ & $\begin{array}{c}\text { No } \\
\text { impactación } \\
\text { Media (DE) }\end{array}$ & Valor p ${ }^{\dagger}$ \\
\hline Ángulo Alfa & $29,7(10,2)$ & $9,8(8,8)$ & $<0,001$ \\
\hline Ángulo Beta & $38,5(12,9)$ & $19,1(9,0)$ & $<0,001$ \\
Distancia & $20,9(4,0)$ & $18,1(4,2)$ & 0,001 \\
\hline
\end{tabular}

+ Valor p de prueba U de Mann Whitney.

Fuente: Autores.

\section{DISCUSIÓN}

Determinar la impactación temprana del canino maxilar es uno de los objetivos primarios al realizar un examen del desarrollo de la oclusión en dentición mixta y permanente para evitar las graves complicaciones que esta puede ocasionar. La impactación afecta la autoestima de los pacientes al alterar la estética y función dental, además disminuye el desarrollo de los maxilares por falta de crecimiento transversal al erupcionar el canino, y causa daño en la simetría de línea media y dientes adyacentes como la reabsorción radicular del incisivo lateral [8].

Por tal motivo, esta investigación buscó la identificación temprana de impactación de caninos maxilares por medio de radiografías panorámicas de las Clínicas de la Universidad Santo Tomás; con el trabajo se buscó mejorar la capacidad diagnóstica de los odontólogos generales, ya que el diagnóstico radiográfico de la impactación de caninos maxilares requiere de un buen análisis para realizar a tiempo el correcto tratamiento. En el presente trabajo el 10,8\% de los caninos evaluados evidenció riesgo de impactación, lo que muestra la alta prevalencia y la necesidad de formación para hacer este diagnóstico de manera oportuna. La mayoría de los pacientes con este riesgo se presentaron en el segundo periodo transicional; edad promedio 9,7 años; lo que nos indica que un tratamiento oportuno según las guías establecidas puede mejorar esta condición; para que el diente pueda erupcionar en la cronología normal entre los 11 y 13 años.

En cuanto al total de pacientes evaluados en el presente estudio el $16,9 \%$ presentaron al menos un canino maxilar con riesgo de impactación. En un estudio realizado por Celikoglu y col [10] la frecuencia de pacientes con caninos maxilares impactados fue $4,9 \%$. En el estudio realizado por Alejos y col [1] se obtuvo una prevalencia en caninos maxilares impactados permanentes de 5,64\% y en otro realizado por Neira se obtuvo una prevalencia de 1,7\% [11]. Estas diferencias se deben reconocer porque este estudio se refiere a riesgo de impactación, no a diagnóstico definitivo de impactación; por lo que sería interesante con los datos obtenidos hacer un seguimiento con control clínico y radiográfico; para determinar qué exactitud tuvo la determinación de riesgo con el diagnóstico definitivo de impactación; aunque es claro hoy en día los pacientes diagnosticados con riesgo deben tratarse de manera interceptiva con las guías de manejo actuales para evitar esa impactación; las guías incluyen exodoncias de caninos temporales; tratamientos de expansión o distalización; y exodoncias de primeros premolares con manejo adecuado de espacios [8-9]. Se resalta de nuevo el factor de prevención para evitar la impactación de 
caninos y cabe destacar que los caninos reportados en este estudio están en la posición 1 y con dos tercios de formación radicular, lo que tendría relación con un mejor pronóstico para el tratamiento de manera interceptiva.

En cuanto la presencia de impactación del canino maxilar según el género, un estudio realizado para identificar la predicción temprana de impactación canina maxilar [12] reveló que hay una incidencia dos veces mayor en mujeres que en hombres. También un estudio de Machado indicó que esta alteración es dos veces más común en mujeres que en hombres [13]. Sin embargo, el presente estudio aunque encontró mayor porcentaje de mujeres con riesgo de impactación $(55,1 \%)$, no mostró diferencias estadísticamente significativas entre hombres y mujeres para presentar riesgo de impactación $(\mathrm{p}=0,853)$; resultados similares fueron reportados por Dutú [14] que tampoco halló diferencias estadísticamente significativas, lo cual, para ambos casos puede ser por el tamaño de la muestra utilizada; en este estudio la muestra solo fue de 171 radiografías, por lo que es conveniente continuar con el estudio y ampliar la muestra.

La edad también se debe tener en cuenta cuando se estudia el riesgo de impactación, en este estudio se encontró un promedio de edad entre 9-10 años para los niños que presentaron riesgo de impactación, al igual que en un estudio realizado por Paz [15], el cual reveló que los niños de 9-10 años presentaron el 62\% de riesgo de impactación del canino. Por otra parte, Dutú [14] afirmó que el intervalo donde se encontró mayor número de diagnósticos definitivos de impactación de caninos; fue entre los 15 a 25 años. Aquí es importante señalar la importancia del diagnóstico temprano, que se considera una ventaja del presente estudio, ya que la edad promedio de erupción del canino maxilar es de los 11 a 13 años y si se hace un diagnóstico como en este estudio entre los 9 a 10 años, se tendrá el tiempo adecuado para facilitar la erupción del canino y evitar las complicaciones; entre más temprano se realice el diagnóstico se contará con más tiempo y mejor pronóstico para el tratamiento ortodóntico de tipo interceptivo.

La ayuda diagnóstica utilizada en esta investigación fue la radiografía panorámica, ya que esta es de uso rutinario por parte de los estudiantes de las clínicas de la Universidad y todos los pacientes en dentición mixta que fueron atendidos contaban con esta ayuda diagnóstica; pero existen reportados diferentes métodos para analizar los riesgos de impactación basados en tomografías y radiografías oclusales. Para este estudio, al igual que los realizados por Alquerban y col y por Alejos y col $[1,9,12]$ se tuvo en cuenta el método de Ericson y Kurol para determinar la impactación de los caninos maxilares con 4 mediciones en las radiografías panorámicas: medición del ángulo alfa, ángulo $\beta$, distancia de la cúspide del canino con el plano oclusal y, finalmente, posición del canino [9].

Al comparar los estudios mencionados, se encontró discrepancia en el punto de corte elegido para definir impactación a partir del ángulo alfa, ya que, en el presente estudio se determinó que obtener un ángulo mayor $25^{\circ}$ indica impactación [9]. En el estudio de Alquerban y col el ángulo alfa elegido fue mayor a $31^{\circ}$ [12], y para el estudio de Alejos y col un ángulo alfa mayor a $65^{\circ}$ indicó una probabilidad mayor de impactación vestibular de los caninos maxilares. Sin embargo, otro predictor importante de combinar con las mediciones de estos ángulos es la distancia del canino al plano oclusal; Sajnani y King [16] encontraron que más allá de los 9 años, la distancia entre la punta de la cúspide canina y el plano maxilar oclusal era el predictor más importante de impactación, más que todas las otras mediciones en radiografías panorámicas, eso se reflejó y coincide en el presente estudio, en el cual se obtuvo una diferencia estadísticamente significativa en la distancia de los caninos con riesgo de impactación, siendo mayor para estos que para los no impactados.

En cuanto la posición dental, un estudio realizado por Warford y col mostró que el $82 \%$ de los caninos impactados se encontraron en la posición 2,3 y 4 [17], Lindauer y col [18] encontraron que el 78\% de los dientes impactados ocurren en la posición 2,3 y 4 , esto contrasta con el presente estudio que reveló que dientes con riesgo de impactación se encontraban principalmente en posición 1. Sin embargo, en este trabajo no solo se consideró esta variable si no también la angulación con respecto a la línea media, la que determinó que, aunque estén en posición 1, muestran riesgo de impactación.

En este estudio de riesgo temprano de impactación de caninos maxilares, se reconoce como limitaciones el tamaño reducido de la muestra y la falta de examinación clínica de los pacientes, que sería una variable 
importante por considerar en el momento de la realización de un estudio longitudinal. De igual manera, aunque la radiografía panorámica es de utilidad para realizar este análisis, con la implementación de estudios con tomografías se lograría analizar mejor las condiciones y riesgos de impactación, así como también las posibles complicaciones.

Como conclusión final, resaltamos la importancia de la radiografía panorámica como medio diagnóstico para identificar riesgo temprano de impactación de caninos; que en el presente estudio fue de $10.8 \%$, tomando como base las mediciones de Erickson y Kurol; donde las variables ángulo alfa y beta y distancia de cúspide de canino a plano oclusal maxilar mostraron tener significancia clínica estadística para determinar este riesgo; por lo que se recomienda estas mediciones sean tenidas en cuenta siempre que se valora la guía de erupción de los caninos maxilares en una radiografía panorámica en paciente de dentición mixta.

\section{AGRADECIMIENTOS}

Queremos agradecerle al Centro de Imágenes Diagnósticas de la Universidad Santo Tomás por proporcionarnos las radiografías panorámicas para realizar las mediciones y llevar a cabo con éxito esta investigación.

\section{REFERENCIAS}

[1] Alejos-Montante K, Martínez-Zumarán A, Torre-Delgadillo D, Rosales-Berber M, Garrocho-Rangel A, Pozos-Guillén A. Early identification of permanent maxillary canine impaction: A radiographic comparative study in a Mexican population. J Clin Exp Dent. 2019;11(3):e282-e286. DOI: 10.4317/jced.55285

[2] Marbán Galán B. La impactación del canino permanente maxilar: estudio prospectivo en un grupo de pacientes ortodóncicos. [Tesis]. Universidad Complutense de Madrid; 2004.

[3] Cardoso-Castro F. Análise e localização da inclinação dos caninos impactados em ortopantomografias. [Trabajo de grado]. Instituto Universitário de Ciências da Saúde; 2017.

[4] Llanera-Peña C. Reabsorciones radiculares: tipos, causas y manejo. Ciencia y Clínica. Gac Dent. 2013;247:114-128.

[5] Camarena-Fonseca A, Rosas-Gonzáles E, Cruzado-Pimichumo L, Liñán-Durán C. Métodos de diagnóstico imagenológico para optimizar el plan de tratamiento y pronóstico de caninos maxilares. Rev Estomatológica Hered. 2017;24(1):263-70.

[6] Pérez-Flores M, Pérez-Flores P, Fierro-Monti C. Alteraciones en la erupción de caninos permanentes. Int J Morphol. 2009;27(1):139-43. DOI: 10.4067/ S0717-95022009000100025.

[7] Upegui-Zea J, González E, Milena D, Ramírez-Ossa D, Restrepo-Narváez L. Determinación del pronóstico en pacientes que presentan caninos maxilares impactados de la Facultad de Odontología de la Universidad de Antioquia. Rev Fac Odontol Univ Antioq. 2009;21(1):75-85. ISSN 0121-246X.

[8] Ericson S, Kurol J. Resorption of maxillary lateral incisors caused by ectopic eruption of the canines. Am J Orthod Dentofacial Orthop. 1988;94(6):503-13. DOI:10.1016/0889-5406(88)90008-x.

[9] Alqerban A, Jacobs R, Fieuws S, Willems G. Radiographic predictors for maxillary canine impaction. Am J Orthod Dentofacial Orthop.2015;147(3):345-54. DOI: 10.1016/j.ajodo.2014.11.018

[10] Celikoglu M, Kamak H, Oktay H. Investigation of transmigrated and impacted maxillary and mandibular canine teeth in an orthodontic patient population. J Oral Maxillofac Surg. 2010;68(5):1001-6. DOI: 10.1016/j. joms.2009.09.006.

[11] Neira-Unda N. Impactación canina maxilar en pacientes clase III esqueletal a causa del déficit de desarrollo maxilar. [Trabajo de grado]. Universidad Andrés Bello. 2014.

[12] Alqerban A, Storms A, Voet M, Fieuws S, Willems G. Early prediction of maxillary canine impaction. Dentomaxillofacial Radiol. 2016;45(3). DOI:10.1259/ dmfr.20150232.

[13] Cruz RM. Orthodontic traction of impacted canines: Concepts and clinical application. Dent Press J Orthod. 2019;24(1):74-87. DOI: 10.1590/2177-6709.24.1.074-087. bbo.

[14] Dutú-Muzás A. Estudio epidemiológico de las retenciones dentarias en una muestra de 2000 pacientes. [Trabajo de grado]. Universidad complutense de Madrid; 2012.

[15] Paz-Salazar I. Prevalencia de caninos superiores retenidos e impactados en pacientes de 9 a 18 años de edad que acuden al centro de atención odontológica de la Universidad de las Américas. [Trabajo de grado]. Universidad de las Américas; 2018.

[16] Sajnani AK, King NM. The sequential hypothesis of impaction of maxillary canine - A hypothesis based on clinical and radiographic findings. J Craniomaxillofacial Surg. 2012;40(8):e375-85. DOI: 10.1016/j.jcms.2012.02.004. 
[17] Warford J, Grandhi R, Tira D. Prediction of maxillary canine impaction using sectors and angular measurement. Am J Orthod Dentofacial Orthop.2003;124(6):651-5. DOI: 10.1016/s0889-5406(03)00621-8.
[18] Lindauer S, Rubenstein L, Hang W, Andersen W, Isaacson R. Canine impaction identified early with panoramic radiographs. J Am Dent Assoc. 1992;123(3):91-7. DOI: 10.14219/jada.archive.1992.0069.

\section{Correo de autores}

María Camila Olaciregui Hernández: maria.olaciregui@ustabuca.edu.co Gabriela Florez Bayona: gabriela.florez@ustabuca.edu.co

Luisanna Fernández Faverola: luisanna.fernandez@ustabuca.edu.co

María Camila Castellanos Rodríguez: maria.castellanos01@ustabuca.edu.co

Adriana Peñuela Sánchez: adriana.penuela@ustabuca.edu.co

Ethman Ariel Torres Murillo: ethman.torres@ustabuca.edu.co 